\title{
Adaptación y validación del cuestionario Estilo de Vida Personal en población joven española
}

\author{
Adaptation and validation of the Personal Lifestyle Questionnaire in \\ young Spanish population
} \begin{abstract}
Pedro Ángel Palomino Moral, ${ }^{4}$ Rafael del Pino Casado ${ }^{5}$
Universidad de Jaén, Jaén, España ${ }^{124}$

Centro de Salud Son Pisa, Palma de Mallorca, España ${ }^{3}$

iD ORCID ID: https://orcid.org/0000-0001-7860-5622 1

ORCID ID: https://orcid.org/0000-0001-5286-9603²

ORCID ID: https://orcid.org/0000-0002-2765-5811 3

ORCID ID: https://orcid.org/0000-0002-8932-2852

ORCID ID: https://orcid.org/0000-0003-2263-2415
\end{abstract}

Henrique Da Silva Domingues, ${ }^{1}$ Antonio Frías Osuna, ${ }^{2 a}$ Vega-Martínez, ${ }^{3}$ María del Carmen

Recibido: 15 de junio de 2021

Aceptado: 05 de octubre de 2021

\section{Resumen}

El objetivo de esta investigación es la adaptación y validación del Cuestionario Estilo de Vida Personal en población joven española de forma diferenciada en chicas y chicos. El estudio de validación se realizó en 827 estudiantes matriculados en la Universidad de Jaén, España. Se analizó la fiabilidad del Cuestionario de Estilo de Vida Personal para población joven (PLQJóvenes) con el análisis de los ítems mediante el coeficiente de correlación de Pearson y coeficiente de correlación intraclase (CCI). La consistencia interna por medio de alfa de Cronbach de la escala total para chicos y chicas y la estabilidad test-retest con el CCI. La validez de constructo se realizó con el análisis factorial exploratorio y la técnica de grupos conocidos. $\mathrm{El}$ alfa de Cronbach para consistencia interna fue de 0,691 para los chicos y de 0,606 para las chicas. El CCI para la estabilidad test-retest fue de 0,823 para las chicas y de 0,956 para los chicos. El análisis factorial exploratorio reveló 5 factores. Se han encontrado asociaciones estadísticamente significativas en las puntuaciones del PLQ entre los subgrupos de SOC (alto y bajo SOC) tanto en chicas como en chicos. En conclusión, el presente estudio puede ser utilizado en investigaciones dirigidas a la identificación de las conductas relacionadas con la salud de los y las jóvenes y también para determinar una puntuación relacionada con el nivel de estilo de vida saludable.

Palabras clave: Estilo de vida, promoción de la salud, Cuestionario de Estilo de Vida Personal, vida saludable, jóvenes 


\begin{abstract}
The objective of this research is the adaptation and validation of the Personal Lifestyle Questionnaire in young Spanish population, in a differentiated way in girls and boys. Validation study carried out with 827 students enrolled at the University of Jaén, Spain. The reliability of the Personal Lifestyle Questionnaire for the young population (PLQ-Young) was analyzed with the analysis of the items using Pearson coefficient and intraclass correlation coefficient (ICC), the internal consistency by means of Cronbach's alpha of the total scale for boys and girls, and test-retest stability with the ICC. Construct validity was performed with the exploratory factor analysis and the known-groups technique. Cronbach's alpha for internal consistency was 0.691 for boys and 0.606 for girls. The ICC for test-retest stability was 0.823 for girls and 0.956 for boys. The exploratory factor analysis revealed 5 factors. Statistically significant associations have been found in PLQ scores between SOC subgroups (high and low SOC) in both boys and girls. In conclusion, the present study can be used in research aimed at identifying behaviors related to the health of young people and also to determine a score related to the level of healthy lifestyle.
\end{abstract}

Keywords: Lifestyle, health promotion, Personal Lifestyle Questionnaire, healthy life, youth

\title{
Introducción
}

En la actualidad, los estilos de vida (EdV) constituyen uno de los determinantes más importantes para la salud de la población (Moral et al. 2018). Para Cockermam (2005) los EdV son patrones de comportamiento colectivos, formados por distintas preferencias que hacen los individuos, relacionados con el contexto y las oportunidades de la vida; incluyen comportamientos que pueden reducir el riesgo de enfermedad, como controlar y gestionar la tensión y las emociones negativas, ocio saludable y uso del tiempo, buenos hábitos de sueño, alimentación y actividad física, evitación del abuso de alcohol, uso de cafeína, tabaco y sustancias psicoactivas; además del sexo seguro; y autocuidados en salud, entre otros.

Por otro lado, la adolescencia y la juventud son etapas de cambios considerables en términos de EdV. El ingreso a la universidad puede dar lugar a nuevas relaciones sociales y al inicio de comportamientos que pueden determinar su estado de salud actual y de por vida. (Aceijas et al., 2017; Ariza et al., 2014; Sánchez-Ojeda, 2015). Por lo tanto, en esta etapa evolutiva y madurativa, conocer los EdV relacionados con la salud es fundamental para el desarrollo de acciones de Promoción de Salud (PS) y la mejora de la calidad de vida (BennasarVeny, 2020; Martínez-Riera et al., 2018).

Cierto número de estudios han destacado la relación entre roles de género y los EdV, mostrando diferencias en el consumo diario de alcohol y tabaco (Fernández-Artamendi et al., 2013; Romo et al., 2015; Simarro et al., 2019) o la actividad física (Sanaeinasab et al., 2013). No obstante, son escasos los instrumentos disponibles en español que valoren de forma global y exclusiva los EdV en la población joven (Leyton et al., 2018). La mayoría de las 
investigaciones utilizan cuestionarios que miden separadamente diferentes $\mathrm{EdV}$, como por ejemplo los hábitos alimentarios (Latzer et al., 2019) o el consumo de sustancias (Grevenstein et al., 2016). En este caso, el estudio de Benassar (2012) valida un cuestionario para jóvenes universitarios, pero además de los EdV, incluye variables sobre el estado de salud percibido, calidad de vida, enfermedad y discapacidad. En el mismo sentido, Grimaldo (2015) desarrolló un cuestionario que también incluía otras variables como las actividades lúdicas y la satisfacción académica.

Disponer de una herramienta para determinar los EdV relacionados con la salud en población joven puede ser útil en la práctica de la Salud Pública, al facilitar el proceso de valoración. Entre los cuestionarios que miden de forma global los EdV se encuentra el Cuestionario de Estilo de Vida Personal (en inglés: Personal Lifestyle Questionnaire - PLQ), desarrollado por Brown et al. (1983). Este instrumento evalúa las prácticas de salud positivas y relevantes y está diseñado para evaluar la capacidad de las personas en adoptar conductas de salud positivas (Mahon et al., 2002). La evidencia psicométrica inicial fue determinada en población adulta (Brown et al., 1983), siendo más tarde validado en adolescentes (Mahon et al., 2002, 2003). Además, el cuestionario PLQ ha sido utilizado en diversos estudios para la medición de los EdV (Trankle, 2009; Atkins, 2017; Yarcheski et al., 2004). En la actualidad no existe una versión validada de dicho cuestionario en España ni en castellano.

Ante esta situación, producir conocimientos sobre EdV relacionados con la salud en los jóvenes es una necesidad (Sánchez-Ojeda, 2015) y se basa en instrumentos fiables y válidos. Por ello, el objetivo de esta investigación es la adaptación y validación del cuestionario PLQ en población joven española (PLQ-Jóvenes), de forma diferenciada en chicas y chicos.

\section{Material y métodos}

El diseño de esta investigación consiste en un estudio de validación de la versión adaptada del cuestionario PLQ en población joven española. La población de estudio consistió en estudiantes de grado de la Universidad de Jaén, matriculados en el curso 2017-2018. Se empleó un muestreo estratificado uniforme seguido de un muestreo aleatorio simple. En primer lugar, se establecieron cuatro estratos de población por rama de estudios: estrato 1: ingenierías; estrato 2: ciencias de la salud y ciencias experimentales; estrato 3: ciencias sociales y jurídicas; y estrato 4: trabajo social, humanidades y ciencias de la educación. Se identificaron los gruposclases de todas las titulaciones de cada estrato. En cada uno de los estratos se seleccionaron los grupos-clases necesarios mediante muestreo aleatorio simple. 
La muestra finalmente obtenida fue de 827 estudiantes, la cual cumple con en tamaño requerido para la realización del análisis más exigente realizado, que es el análisis factorial exploratorio. Además, el estudio recibió la aprobación del Comité de Ética de la Investigación de la Universidad de Jaén.

\section{Descripción del cuestionario PLQ}

El cuestionario PLQ, en su formato original, consta de 24 ítems para mujeres y 23 para hombres (el ítem 22 es solo para mujeres). Los ítems hacen referencia a seis áreas (Brown et al., 1983): nutrición, ejercicio, relajación, seguridad, uso de sustancias y promoción de la salud. Cada ítem se contesta con una escala tipo Likert del 1 (nunca) al 4 (siempre), siendo el rango de puntuaciones de 24 a 96 para mujeres y de 24 a 92 para hombres (directamente proporcional a las conductas positivas). Se deben revertir las puntuaciones de 8 ítems (7r, 13r, 14r, 16r, 17r, 20r, 21r, 28r) para el cómputo global del cuestionario.

El cuestionario ha mostrado aceptables índices de consistencia interna en adolescentes (entre 0,72 y 0,80) (Mahon et al., 2002, 2003). En cuanto a la validez de contenido, algunos autores afirman que los ítems construidos para el PLQ implican acciones y comportamientos que son considerados en la literatura como los más prevalentes para el cuidado personal de la salud (Brown et al., 1983).

\section{Proceso de adaptación y prueba piloto (cuestionario PLQ-Jóvenes)}

\section{Adaptación lingüística y cultural}

Los ítems del cuestionario PLQ fueron traducidos del inglés al español y, posteriormente, retraducidos al inglés por dos investigadores y un profesor de nacionalidad británica de forma independiente, consensuando posteriormente las discrepancias. La versión en español fue revisada por un grupo de expertos en PS en jóvenes, con la finalidad de mejorar su redacción y valorar la posible inclusión de nuevos ítems.

\section{$\underline{\text { Prueba piloto }}$}

Con el cuestionario resultante (PLQ-Jóvenes) se realizó una prueba piloto sobre 20 estudiantes (10 chicas y 10 chicos) a fin de valorar el grado de comprensión de los ítems y el tiempo necesario para contestar el cuestionario.

\section{Validación}

La validación del cuestionario PLQ-jóvenes incluyó los procesos que a continuación se detallan de forma separada para chicos y chicas. 


\section{$\underline{\text { Recolección de datos }}$}

La recogida de datos incluyó un apartado de características sociodemográficas, el cuestionario PLQ-Jóvenes y la escala Orientation to Life Questionnaire (OLQ-13) (Lundberg y Peck, 1995), utilizada para la validez de constructo. La recolección de datos se llevó a cabo en marzo y abril del 2018 en los grupos-clases seleccionados. Previamente a la recogida de datos, se explicó el objetivo y procedimiento de la investigación a los participantes, haciendo énfasis en el carácter voluntario de dicha participación y la confidencialidad de todo el proceso de investigación y de sus resultados. Además, se procedió a la obtención del consentimiento informado. No hubo ningún rechazo a participar en el estudio.

\section{$\underline{\text { Análisis estadístico }}$}

Para determinar la fiabilidad del cuestionario hemos utilizado las siguientes técnicas estadísticas:

1) Análisis de los ítems. Se analizó la correlación entre ítems y de cada uno de ellos con la puntuación total de la escala mediante el coeficiente de correlación de Pearson.

2) Consistencia interna. Se ha calculado el coeficiente alfa de Cronbach para la escala total y para cada dimensión, tomando las consideraciones de Maroco y García-Marqués (2006), en las que indican que la consistencia interna con un $\alpha$ de 0,60 se considera admisible, siempre que los resultados que se obtengan se interpreten con cautela y tengan en consideración el contexto. 3) Estabilidad. La fiabilidad test-retest se midió con el CCI. Se administró el cuestionario PLQJóvenes con un intervalo de 2 semanas a un grupo de 41 estudiantes de todas las titulaciones, cursos y género, seleccionados por conveniencia.

4) Validez de constructo. Se examinó mediante análisis factorial exploratorio (AFE). Siguiendo las recomendaciones de Costello y Osborne (2005) para las características de los datos del presente estudio (ausencia de normalidad en las puntuaciones de los ítems y correlación entre ítems de baja a mediana), el AFE se realizó mediante extracción de factorización de ejes principales y rotación Varimax. Para el número de factores a seleccionar se utilizó el gráfico de sedimentación, siguiendo también las recomendaciones de Costello y Osborne (2005).

Por otra parte, se evaluó la validez de constructo mediante la técnica de grupos conocidos. Según estudios previos, se ha encontrado relación entre los EdV y Sentido de Coherencia (SOC) (Martinez et al., 2018; Nilsen et al., 2015; Sahlen et al., 2013). Para la medición del SOC se utilizó la escala OLQ-13, la cual se compone de tres elementos: la comprensibilidad, que se trata del grado en que los individuos disponen de un sentido cognitivo 
de los estímulos que tienen en el presente y en el futuro; la manejabilidad, que es el grado en que los individuos entienden que los recursos adecuados para hacer frente a las demandas del medio se encuentran a su disposición; y la significatividad, que se refiere al valor que el sujeto atribuye a lo que sucede, independientemente de la forma en que se produzca (Antonovsky, 1996).

La escala OLQ-13 presenta un nivel de consistencia alto, con rangos del alfa de Cronbach que van de 0,70 a 0,92. Los ítems son medidos por una escala Likert de 1 a 7 con una puntuación mínima de 13 y máxima de 91 puntos (Eriksson \& Lindström, 2005). Se utilizó la mediana como punto de corte para definir la puntuación de SOC alto o bajo. Se analizó la diferencia de medias de PLQ-Jóvenes entre los subgrupos de SOC mediante la t de Student y se determinó el tamaño de efecto mediante la d de Cohen. Los análisis se realizaron con el programa SPSS Statistics 23. El estudio recibió la aprobación del Comité de Ética de la Investigación de la Universidad de Jaén.

\section{Resultados}

\section{Características socio-demográficas de la muestra}

Las características socio-demográficas de la muestra se exponen en la Tabla 1. La muestra estaba compuesta por 827 estudiantes, de los que $286(34,60 \%)$ son chicos y $541(65,40 \%)$ son chicas. La edad media fue de 21,1 (desviación estándar: 4,03).

\section{Adaptación del cuestionario PLQ-Jóvenes y prueba piloto}

En el proceso de traducción del inglés al español y la retraducción no se encontraron discrepancias destacables. De la versión en español del cuestionario, un grupo de expertos e investigadores en PS realizó adaptaciones culturales de los ítems 9, 12, 13 y 20, con el objetivo de aumentar la comprensibilidad. Adicionalmente, se decidió la inclusión de los ítems 17 (consumo de alcohol), 26 (uso de casco de seguridad), 27 (relaciones sexuales) y 28 (consumo de drogas ilegales) de forma que el cuestionario final PLQ-Jóvenes está compuesto por 28 ítems para chicas y 27 para chicos, siendo el rango teórico de puntuaciones de 28 a 112 y de 27 a 108 , respectivamente. La prueba piloto puso de manifiesto una adecuada comprensión del cuestionario por parte de los participantes y un tiempo de cumplimentación aceptable, por lo que no se hicieron modificaciones en el cuestionario tras dicha prueba piloto. 


\section{Tabla 1}

Características socio-demográficas

\begin{tabular}{cccc}
\hline VARIABLE & & $\mathbf{N}$ & $\mathbf{\%}$ \\
\hline GÉNERO & MASCULINO & 286 & 34,6 \\
& FEMENINO & 541 & 65,4 \\
NIVEL DE ESTUDIO DEL & SIN ESTUDIO & 113 & 13,7 \\
PADRE & PRIMARIA & 318 & 38,5 \\
& SECUNDARIA & 270 & 32,6 \\
& UNIVERSITARIOS & 122 & 14,8 \\
NIVEL DE ESTUDIO DE LA & NS/NC & 4 & 0,5 \\
MADRE & SIN ESTUDIO & 113 & 13,7 \\
& PRIMARIA & 330 & 39,9 \\
& SECUNDARIA & 276 & 33,4 \\
& UNIVERSITARIOS & 104 & 12,6 \\
& NS/NC & 4 & 0,5 \\
\hline
\end{tabular}

\section{Descripción de los resultados del cuestionario}

El tiempo medio para responder a las preguntas del cuestionario fue de 9 minutos. La puntuación media para las chicas fue de 85,16 (rango: 65 - 105) con una desviación estándar de 6,60. Para los chicos la media fue de 82,88 (rango: 58 - 106) con una desviación estándar de 7,74. Estas diferencias fueron estadísticamente significativa ( $<0,001)$ (Tabla 2).

\section{Tabla 2}

Diferencias de género en las puntuaciones del PLQ-Jóvenes

\begin{tabular}{|c|c|c|c|c|c|}
\hline FACTOR & CHICOS & CHICAS & Valor de $\mathbf{P}$ & d de Cohen & IC \\
\hline \multirow{4}{*}{$\begin{array}{c}\text { TOTAL PLQ - Jóvenes } \\
\text { Conductas saludables en actividad } \\
\text { física }\end{array}$} & $\mathrm{N}=286$ & $N=541$ & & & \\
\hline & $\begin{array}{c}\text { Media y desviación } \\
\text { estándar }\end{array}$ & $\begin{array}{l}\text { Media y desviación } \\
\text { estándar }\end{array}$ & & & \\
\hline & $82,88+/-7,742$ & $85,16+/-6,601$ & $<0,001$ & $(-) 0,32$ & $(-) 0,46 ;(-) 0,19$ \\
\hline & $11,06+/-3,055$ & $10,02+/-2,645$ & $<0,001$ & 0,37 & 0,$23 ; 0,51$ \\
\hline $\begin{array}{c}\text { Conductas relacionadas con el consumo } \\
\text { de alcohol y seguridad }\end{array}$ & $20,98+/-2,936$ & $21,93+/-2,586$ & $<0,001$ & $(-) 0,35$ & $(-) 0,49 ;(-) 0,21$ \\
\hline $\begin{array}{c}\text { Conductas saludables en descanso y } \\
\text { bienestar }\end{array}$ & $9,26+/-1,863$ & $8,53+/-1,870$ & $<0,001$ & 0,39 & 0,$25 ; 0,53$ \\
\hline $\begin{array}{l}\text { Otras conductas de prevención y } \\
\text { promoción para la salud }\end{array}$ & $30,30+/-3,772$ & $33,27+/-3,372$ & $<0,001$ & $(-) 0,84$ & $(-) 0,70 ;(-) 0,99$ \\
\hline $\begin{array}{l}\text { Conductas relacionadas con el consumo } \\
\text { de otras sustancias }\end{array}$ & $11,28+/-1,603$ & $11,41+/-1,239$ & 0,236 & $(-) 0,09$ & $(-) 0,23 ;(-) 0,04$ \\
\hline
\end{tabular}

Nota: Total y por dimensiones. Categoría de interés: chicos

\section{Validación}

1) Análisis de los ítems. Las correlaciones entre cada ítem del cuestionario PLQ-Jóvenes y la puntuación total de las chicas fue significativas en todos los ítems, siendo la correlación más 
alta 0,447 y la más baja 0,134; el rango de las correlaciones de los ítems entre sí osciló entre 0,002 y 0,795 . Para los chicos, las correlaciones entre los distintos ítems y el resultado de la puntuación total también fueron significativos en todos los ítems, siendo la correlación más alta 0,513 y la más baja 0,154 ; el rango de las correlaciones de los ítems entre si osciló entre 0,004 y 0,833 .

2) Consistencia interna. El alfa de Cronbach encontrada es de 0,691 para chicos y 0,606 para chicas.

3) Estabilidad. El CCI para la fiabilidad test-retest fue de 0,823 (IC 95\%: 0,598; 0,922) para las chicas y de 0,956 (IC 95\%: 0,316; 0,997) para los chicos.

4) Validez de constructo. De acuerdo con el gráfico de sedimentación (Figura 1), en el AFE identificamos 5 factores, que explicaron el 36,77\% de la varianza acumulada (Tabla 3). El factor 1, denominado "conductas saludables en actividad física", explicó un 10,32\% de la varianza. El factor 2, "conductas relacionadas con el consumo de alcohol y seguridad", explicó un 9,71\%. El factor 3, denominado "conductas saludables en descanso y bienestar", explicó un 6,32\%. El factor 4, "otras conductas de prevención y promoción para la salud", explicó un 5,95\% y el factor 5, "conductas relacionadas con el consumo de otras sustancias", explicó un 4,45\% del total de la varianza. Los valores del alfa de Cronbach para cada una de los cinco factores resultantes del análisis factorial oscilaron entre 0,7 y 0,48 (Tabla 3).

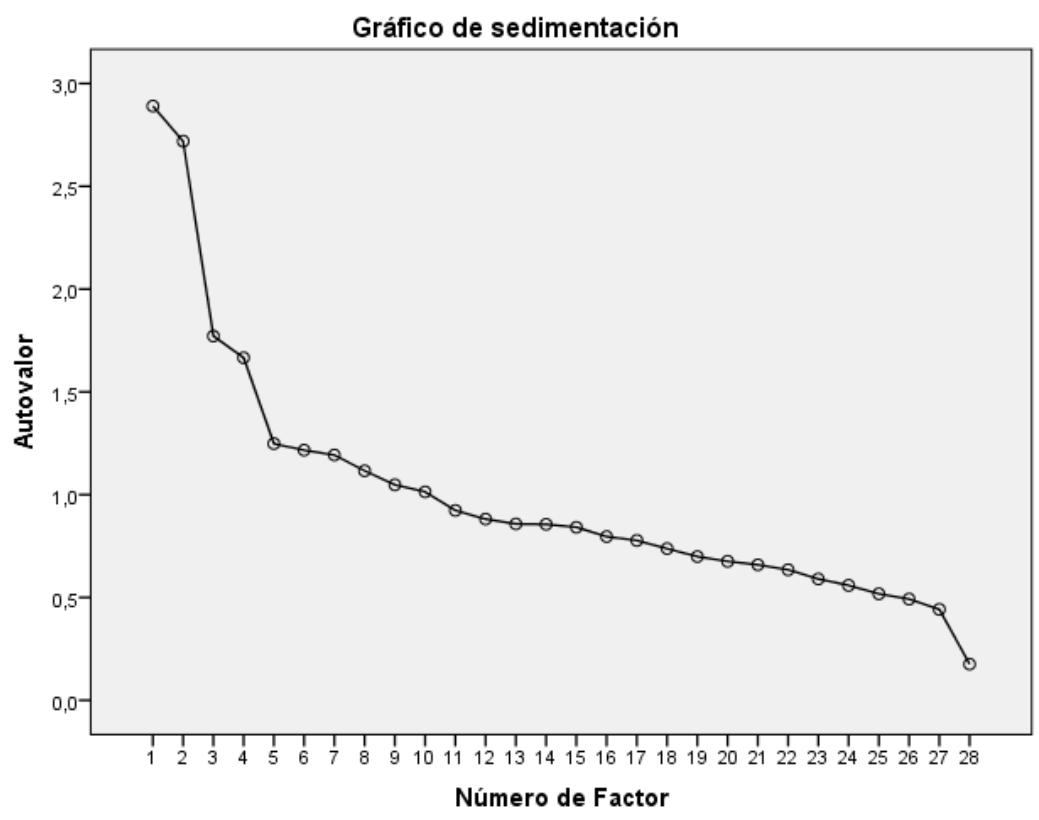

Figura 1. Gráfico de sedimentación 


\section{Tabla 3}

Resultados del análisis factorial exploratorio

\begin{tabular}{|c|c|c|c|c|}
\hline & Nombre & $\alpha$ & Ítems PLQ-Jóvenes & $\%$ de varianza \\
\hline Factor 1 & Conductas saludables en actividad física & 0,70 & $10,11,18,24$ & 10,322 \\
\hline Factor 2 & $\begin{array}{l}\text { Conductas relacionadas con el consumo de } \\
\text { alcohol y seguridad }\end{array}$ & 0,49 & 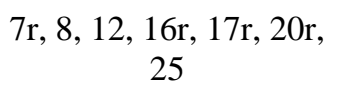 & 9,713 \\
\hline Factor 3 & $\begin{array}{c}\text { Conductas saludables en descanso y } \\
\text { bienestar }\end{array}$ & 0,55 & $9,15,19$ & 6,328 \\
\hline Factor 4 & $\begin{array}{l}\text { Otras conductas de prevención y promoción } \\
\text { para la salud }\end{array}$ & 0,48 & $\begin{array}{c}1,2,3,4,5,6,14 \mathrm{r}, 22 \\
23,26,27\end{array}$ & 5,951 \\
\hline Factor 5 & $\begin{array}{l}\text { Conductas relacionadas con el consumo de } \\
\text { otras sustancias }\end{array}$ & 0,52 & $13 \mathrm{r}, 21 \mathrm{r}, 28 \mathrm{r}$ & 4,458 \\
\hline
\end{tabular}

La pertinencia del AFE se justifica con el resultado del test de esfericidad de Barlett (p $<0,01$ para chicas y chicos) y el índice KMO (0,664 para chicas y 0,680 para chicos). Con respecto a la técnica de grupos conocidos, se encontraron diferencias estadísticamente significativas entre la puntuación media del PLQ-Jóvenes en cada uno de los 2 subgrupos de SOC a favor del grupo de SOC alto ( $<<0,001$ y d de Cohen de 0,50 para las chicas; $p=0,005$ y d de Cohen de 0,34 para los chicos).

\section{Discusión}

En el presente estudio se ha adaptado y validado el cuestionario PLQ en población joven española. Esta nueva versión PLQ-Jóvenes es útil para identificar las conductas y valorar los EdV relacionadas con la salud en jóvenes. Este cuestionario permite determinar la frecuencia de exposición a conductas relacionadas con la actividad física, el consumo de sustancias, alimentación, seguridad, descanso, sexualidad, entre otras. El PLQ-Jóvenes ha sido adaptado y validado de forma independiente para chicos y chicas.

Asimismo, el cuestionario PLQ-Jóvenes para población española permite el estudio de las conductas relacionadas con la salud en este grupo de población y contexto cultural. Hasta el momento solo había sido validado en población adulta (Brown et al., 1983) y adolescente (Mahon et al., 2002, 2003) de Estados Unidos.

Este estudio demuestra que el cuestionario PLQ-Jóvenes reúne aceptables propiedades métricas, tanto en el referente a la fiabilidad como a la validez y tanto para chicas como para 
chicos, siendo estas propiedades métricas similares a las encontradas en la validación del original en adultos (Brown et al., 1983) y en su validación en adolescentes (Mahon et al., 2002, 2003).

Con base en los resultados del AFE, se aceptó una solución de cinco factores que supone una nueva estructura de las dimensiones del cuestionario PLQ propuesta inicialmente por Brown et al. (1983). El primer factor, denominado "conductas saludables en actividad física", demostró una consistencia interna aceptable. Los demás factores alcanzaron menores niveles de confiabilidad. Los resultados del AFE del PLQ-Jóvenes indican la necesidad del uso de este cuestionario de forma global incluyendo el total de ítems, al igual que análisis factoriales previos realizados en adultos (Brown et al., 1983) y adolescentes (Mahon et al., 2002, 2003) en otros contextos. Las diferentes estructuras factoriales encontradas en los estudios de validación realizados, refuerzan la importancia de validar el cuestionario PLQ previo a su uso en diferentes entornos socioculturales.

Los resultados de la técnica de grupos conocidos refuerzan la validez de constructo del cuestionario, poniendo de manifiesto las diferencias de puntuación del PLQ-Jóvenes entre los grupos de SOC alto y bajo, a favor del grupo de SOC alto. De acuerdo con investigaciones previas (Martinez et al., 2018; Nilsen et al., 2015; Sahlen et al., 2013), una mayor puntuación en Sentido de Coherencia se relaciona con EdV más saludables.

Por otro lado, se ha encontrado diferencias estadísticamente significativas con relación al género, de forma que las chicas han obtenido en el cuestionario una puntuación media más alta que los chicos. Las diferencias de género encontradas están en la misma línea de otros estudios en los que se ha abordado también la relación del género con los estilos de vida (Bennasar-Veny, 2020; Gallardo-Escudero et al., 2014). El cuestionario PLQ-Jóvenes, si bien es un único instrumento, se ha validado de forma independiente tanto para chicos como para chicas, obteniendo valores métricos adecuados para ambos. En el caso de las chicas, el cuestionario cuenta con un ítem más que hace referencia a la realización de autoexploración mamaria. El PLQ-Jóvenes permite el estudio de los EdV en población joven permitiendo una evaluación diferenciada en chicos y chicas.

Como limitación de este estudio, hay que destacar que ha sido realizado en población joven universitaria. En principio se podría entender que también sería útil para el resto de la población joven, si bien esto no queda demostrado en este estudio. El estudio ha sido realizado en una única universidad pública. No obstante, es probable que las características sociodemográficas sean similares al resto de la población joven universitaria española. De 
cualquier forma, se hace necesario la validación futura en otras poblaciones de jóvenes universitarios y no universitarios.

\section{Conclusión}

Como conclusión de este estudio se destaca que el cuestionario PLQ-Jóvenes es de fácil y de rápida administración, posee adecuadas propiedades métricas y permite, por una parte, la identificación de las conductas relacionadas con la salud tanto en chicos como en chicas y por otra, determinar una puntuación relacionada con el nivel de estilo de vida saludable.

\section{Referencias}

Aceijas, C., Waldhäusl, S., Lambert, N., Cassar, S., \& Bello-Corassa, R. (2017). Determinants of health-related lifestyles among university students. Perspectives in public health, 137 (4), 227-236. http://dx.doi.org/10.1177/1757913916666875.

Antonovsky, A. (1996). The salutogenic model as a theory to guide health promotion. Health promotion international, 11 (1), 11-18. http://dx.doi.org/10.1093/heapro/11.1.11.

Ariza, C., García-Continente, X., Villalbí, J. R., Sánchez-Martínez, F., Pérez, A., \& Nebot, M. (2014). Consumo de tabaco de los adolescentes en Barcelona y tendencias a lo largo de 20 años. Gaceta Sanitaria, 28, 25-33. http://dx.doi.org/10.1016/j.gaceta.2013.08.005.

Atkins, R. L. (2017). Outcomes of depression in Black single mothers. Clinical nursing research, 26 (4), 464-483. http://dx.doi.org/10.1177/1054773816633440.

Bennasar-Veny M. (2012). Estilos de vida y salud en estudiantes universitarios: la universidad como entorno promotor de la salud [tesis doctoral, Universitat de les Illes Balears].

Bennasar-Veny, M., Yañez, A. M., Pericas, J., Ballester, L., Fernandez-Dominguez, J. C., Tauler, P., \& Aguilo, A. (2020). Cluster analysis of health-related lifestyles in university students. International journal of environmental research and public health, 17 (5), 1776. http://dx.doi.org/10.3390/ijerph17051776.

Brown, N., Muhlenkamp, A., Fox, L., \& Osborn, M. (1983). The relationship among health beliefs, health values, and health promotion activity. Western journal of nursing research, 5 (2), 155-163. http://dx.doi.org/10.1177/019394598300500205.

Cockerham, W. C. (2005). Health lifestyle theory and the convergence of agency and structure. Journal of health and social behavior, 46 (1), 51-67.

http://dx.doi.org/10.1177/002214650504600105 
Costello, A. B., \& Osborne, J. (2005). Best practices in exploratory factor analysis: Four recommendations for getting the most from your analysis. Practical assessment, research, and evaluation, 10 (1), 7.

Eriksson, M., \& Lindström, B. (2005). Validity of Antonovsky's sense of coherence scale: a systematic review. Journal of Epidemiology \& Community Health, 59 (6), 460-466. http://dx.doi.org/10.1136/jech.2003.018085.

Fernández-Artamendi, S., Secades-Villa, R., Fernandez Hermida, J. R., García-Fernández, G., \& García-Rodríguez, O. (2013). Gender differences in early alcohol and tobacco use as a risk factor in Spanish adolescents. Substance use \& misuse, 48 (6), 429-437. http://dx.doi.org/10.3109/10826084.2013.776085.

Gallardo-Escudero, A., MJ, M. A., Planells del Pozo, E. M., \& Aliaga, L. (2014). The university stage does not favor the healthy life style in women students from Granada. Nutricion hospitalaria, 31 (2), 975-979. https://dx.doi.org/10.3305/nh.2015.31.2.8303.

Grevenstein, D., Bluemke, M., \& Kroeninger-Jungaberle, H. (2016). Incremental validity of sense of coherence, neuroticism, extraversion, and general self-efficacy: Longitudinal prediction of substance use frequency and mental health. Health and Quality of Life Outcomes, 14 (1), 1-14. http://dx.doi.org/10.1186/s12955-016-0412-z.

Muchotrigo, M. P. G. (2007). Construcción de un instrumento sobre estilos de vida saludables en estudiantes universitarios. Journal of Psychology, 9 (1), 8-20.

Latzer, Y., Weinberger-Litman, S. L., Spivak-Lavi, Z., \& Tzischinsky, O. (2019). Disordered eating pathology and body image among adolescent girls in israel: the role of sense of coherence. Community Mental Health Journal, 55 (7), 1246-1252. http://dx.doi.org/10.1007/s10597-019-00446-0.

Leyton, M., Lobato, S., Batista, M., Aspano, M. I., \& Jiménez, R. (2018). Validación del cuestionario de estilo de vida saludable (EVS) en una población española. Revista iberoamericana de psicología del ejercicio y el deporte, 13 (1), 23-31. Disponible en: https://www.redalyc.org/articulo.oa?id=311153534002.

Lundberg, O., \& Peck, M. N. (1995). A simplified way of measuring sense of coherence: experiences from a population survey in Sweden. The European Journal of Public Health, 5 (1), 56-59. http://dx.doi.org/10.1093/eurpub/5.1.56.

Mahon, N. E., Yarcheski, A., \& Yarcheski, T. J. (2002). Psychometric evaluation of the personal lifestyle questionnaire for adolescents. Research in nursing \& health, 25 (1), 6875. http://dx.doi.org/10.1002/nur.10017 
Mahon, N. E., Yarcheski, T. J., \& Yarcheski, A. (2003). The revised personal lifestyle questionnaire for early adolescents. Western Journal of Nursing Research, 25 (5), 533 547. http://dx.doi.org/10.1177/0193945903253000

Maroco, J., \& Garcia-Marques, T. (2006). Qual a fiabilidade do alfa de Cronbach? Questões antigas e soluções modernas?. Laboratório de psicologia, 4 (1), 65-90. http://dx.doi.org/10.14417/lp.763.

Martinez, M. D. C. V., Osuna, A. F, \& Casado, R. D. P. (2018). Validity and reliability of the sense of coherence scale among nursing undergraduate students from a Spanish $\begin{array}{llll}\text { university. } & \text { Gaceta } & \text { sanitaria, } & 33\end{array}$ (4), https://doi.org/10.1016/j.gaceta.2018.02.009.

Martínez-Riera, J. R., Pino, C. G., Pons, A. A., Mendoza, M. C. G., López-Gómez, J., \& Acevedo, H. V. A. (2018). La universidad como comunidad: universidades promotoras de salud. Informe SESPAS 2018. Gaceta sanitaria, 32, 86-91. https://doi.org/10.1016/j.gaceta.2018.08.002.

Moral, P. A. P., Gascón, M. L. G., \& Abad, M. L. (2014). La salud y sus determinantes sociales. Desigualdades y exclusión en la sociedad del siglo XXI. Revista internacional de sociología, 72, 45-70. https://doi.org/10.3989/ris.2013.02.16.

Nilsen, V., Bakke, P. S., Rohde, G., \& Gallefoss, F. (2015). Is sense of coherence a predictor of lifestyle changes in subjects at risk for type 2 diabetes?. Public Health, 129 (2), 155 161. http://dx.doi.org/10.1016/j.puhe.2014.12.014.

Avilés, N. R., Marcos, J. M., García, E. G., Márquez, A. M., \& Camacho, A. T. (2015). Bebiendo como chicos: consumo compartido de alcohol y rupturas de género en poblaciones adolescentes. Revista Española de Drogodependencias, 40 (1).

Sahlen, K. G., Johansson, H., Nyström, L., \& Lindholm, L. (2013). Health coaching to promote healthier lifestyle among older people at moderate risk for cardiovascular diseases, diabetes and depression: a study protocol for a randomized controlled trial in Sweden. BMC Public Health, 13 (1), 1-9. http://dx.doi.org/10.1186/1471-2458-13-199.

Sanaeinasab, H., Saffari, M., Nazeri, M., Karimi Zarchi, A., \& Cardinal, B. J. (2013). Descriptive analysis of I ranian adolescents' stages of change for physical activity behavior. Nursing \& health sciences, 15 (3), 280-285. https://doi.org/10.1111/nhs.12020.

Sánchez-Ojeda, M. A. (2015). Healthy lifestyles of the university population. Nutricion hospitalaria, 31 (5), 1910-1919. https://dx.doi.org/10.3305/nh.2015.31.5.8608.

Simarro, J. V. C., Cañigral, F. J. B, Esteban, M. P. P., Gregori, L. P., \& Arévalo Arévalo, G. (2019). Consumo de sustancias adictivas en jóvenes universitarios de la Comunidad 
Valenciana

(España).

Metas

enferm,

14-20.

https://doi.org/10.35667/MetasEnf.2019.22.1003081480.

Trankle, S. A., \& Haw, J. (2009). Predicting Australian health behaviour from health beliefs. Electronic Journal of Applied Psychology, 5 (2), 9-17. http://dx.doi.org/10.7790/ejap.v5i2.167.

Yarcheski, A., Mahon, N. E., Yarcheski, T. J., \& Cannella, B. L. (2004). A meta-analysis of predictors of positive health practices. Journal of Nursing Scholarship, 36 (2), 102-108. http://dx.doi.org/10.1111/j.1547-5069.2004.04021.x. 